\title{
Parametric Study of Fatigue Crack Growth in a Finite Plate
}

\author{
Luís D. C. Ramalho' ${ }^{1}$, Raul D. S. G. Campilho², Jorge Belinha ${ }^{3}$, \\ Paulo M. S. T. de Castro ${ }^{4}$ \\ ${ }^{1}$ INEGI-Institute of Science and Innovation in Mechanical and Industrial Engineering, Rua Dr. \\ Roberto Frias, 4200-465 Porto, Portugal (Iramalho@inegi.up.pt) ORCID 0000-0003-2579- \\ 4057; 2ISEP-School of Engineering, Polytechnic of Porto, Rua Dr. António Bernardino de \\ Almeida, 431, 4200-072 Porto, Portugal (raulcampilho@gmail.com) ORCID 0000-0003-4167- \\ 4434; ${ }^{3}$ SEEP-School of Engineering, Polytechnic of Porto, Rua Dr. António Bernardino de \\ Almeida, 431, 4200-072 Porto, Portugal (job@isep.ipp.pt) ORCID 0000-0002-0539-7057; \\ ${ }^{4}$ Faculty of Engineering, University of Porto, Rua Dr. Roberto Frias, 4200-465 Porto, Portugal \\ (ptcastro@fe.up.pt) ORCID 0000-0003-3202-1343
}

\begin{abstract}
Fatigue crack propagation is an undesirable phenomenon that may lead to catastrophic failures in many components and structures, therefore it is important to understand its underlying mechanics. To that effect, systematic parametric studies of fatigue crack propagation laws are interesting to determine how fatigue life varies with the constants that define the mechanical behavior of a given material in a fatigue situation, such as the Paris' law constants, fracture toughness (Kc) or the stress range $\Delta \sigma$.

The parametric studies performed in the present work assess the influence of several parameters, assuming that failure occurs when $\mathrm{K}>\mathrm{Kc}$, but also when all the material ahead of the crack is yielding. It was found that $m$ and $C$, the Paris' law parameters, are the most influential parameters in terms of fatigue life. The present study should help future designers when choosing materials for components or structures subjected to cyclic loads.
\end{abstract}

Author Keywords. Paris' Law, Fatigue, Fracture, Failure.

Type: Research Article

$\partial$ Open Access $\square$ Peer Reviewed @) CC BY

\section{Introduction}

The durability of components is an increasingly important subject due to the environmental impacts of less durable components that need to be frequently replaced, meaning that more durable structures and components have to be made. However, making a component durable is only part of the problem since, due to limitations in carbon emissions in transport industries, it is also necessary to make components lightweight. So, nowadays engineers have to balance these two factors and try to make components that are durable and lightweight, which can be a challenge.

This is where fatigue mechanics serves as an important tool, emerging in the XIX century (Schütz 1996) with works such as Albert (1838) and Wöhler (1860), among others. According to Stephens et al. (2001) there are four different ways to evaluate fatigue, the stress-life (S-N) model, the strain life $(\varepsilon-\mathrm{N})$ model, the fatigue crack growth model $(d a / d N-\Delta K)$, and the two stage model which combines the strain model and the crack growth model. This work focuses on the fatigue crack growth model, combining Linear Elastic Fracture Mechanics (LEFM) with fatigue by using the stress intensity factor (K), proposed by Irwin (1957), and Paris' Law (Paris, Gomez, and Anderson 1961; Paris and Erdogan 1963). 
The threshold stress intensity factor $\left(\Delta K_{t h}\right)$, and how it is influenced by different parameters, was reviewed by Lawson, Chen, and Meshii (1999). It was concluded that it generally decreases with increasing crack length $(a)$ or stress ratio $(R)$ (Bulloch 1991). Moreover, $\Delta K_{t h}$ tends to decrease as yield stress $\left(\sigma_{Y}\right)$ increases, at least for steels (Ritchie 1979). The $\Delta K_{\text {th }}$ can be considered as a minimum $K$ needed for the crack to grow under fatigue, even if in reality cracks still grow below this value, but at a much reduced rate. This parameter was also considered in the present work.

Parametric studies of Paris' law have been made (Butler and Rodríguez 1992; Mínguez and Vogwell 1993). These studies show how fatigue life changes with variations of several constants characterizing material mechanical behavior, namely the Paris' law constants ( $m$ and $C$ ) and the critical stress intensity factor $\left(K_{c}\right)$. However, those studies do not evaluate the impact of other important parameters like the $\Delta K_{t h}$, the dominant failure mechanism for characterizing critical crack length - fracture or plastic collapse -, or even the various possible forms of the relationship $K=f(a, \ldots)$. These factors led to an interest in performing a study evaluating the parameters that Butler and Rodríguez (1992) and Mínguez and Vogwell (1993) did not consider. Therefore, the present work aims at determining the influence of several material and geometric parameters in the fatigue life using as a case study a finite plate with a central crack.

\section{Methodology}

In a plate under mode I loading the $K$ is determined using the following equation:

$$
\Delta K=Y \Delta \sigma \sqrt{\pi a}
$$

with $Y$ being the geometry factor of the problem, $\Delta \sigma$ is the stress range and $a$ is the half-crack length. In a plane with finite width $(W) Y \neq 1$ and is dependent on $W$ (Mínguez and Vogwell 1993):

$$
Y=\sqrt{\sec \frac{\pi a}{2 W}}=\left(\cos \frac{\pi a}{2 W}\right)^{-1 / 2}
$$

which means that the critical crack length $\left(a_{c}\right)$ can be obtained with the following equation:

$$
\begin{gathered}
K_{c}=\left(\cos \frac{\pi a_{c}}{2 W}\right)^{-1 / 2} \sigma_{\max } \sqrt{\pi a_{c}} \\
\frac{\mathrm{a}_{\mathrm{c}}}{\cos \frac{\pi \mathrm{a}_{\mathrm{c}}}{2 \mathrm{~W}}}=\frac{\mathrm{K}_{\mathrm{c}}^{2}}{\sigma_{\max }^{2} \pi}
\end{gathered}
$$

$\sigma_{\max }$ being the maximum stress applied to the plate. Paris' law, proposed in the early $1960 \mathrm{~s}$ (Paris, Gomez, and Anderson 1961; Paris and Erdogan 1963), relates $\Delta K$ with the crack growth rate as follows:

$$
\frac{\mathrm{da}}{\mathrm{dN}}=\mathrm{C}(\Delta \mathrm{K})^{\mathrm{m}}
$$

For the finite width plate this can be rearranged to write as follows:

$$
d N=\frac{1}{C(\Delta \sigma \sqrt{\pi})^{m}}\left(\frac{\cos \frac{\pi a}{2 W}}{a}\right)^{m / 2} d a
$$

The integration of this function can be performed using a summation, which results in negligible errors given that the chosen crack increment $(\Delta a)$ is small enough. The analysis of fatigue life in a finite plate with a crack can be enhanced by considering that the plate can also fail due to reaching yield in the whole region of material ahead of the crack, grey band in 
Figure 1. Using a simplified approach, the average stress in that region can be defined by the relationship between the plate width and crack length as follows:

$$
\sigma_{a c}=\sigma_{\max } \frac{W}{W-a}
$$

After some transformation, and considering that this stress has to be equal to the $\sigma_{Y}$ of the material to have general yielding causing failure, the crack length at which this occurs $\left(a_{Y}\right)$ can be defined as:

$$
a_{Y}=\frac{\sigma_{Y} W-\sigma_{\max } W}{\sigma_{Y}}
$$

Similarly to $K_{c}, \Delta K_{\text {th }}$ can be defined as:

$$
\Delta K_{t h}=\left(\cos \frac{\pi a_{t h}}{2 W}\right)^{-1 / 2} \Delta \sigma \sqrt{\pi a_{t h}}
$$

This can then be transformed to find the minimum crack length to exceed $\Delta K_{t h}\left(a_{t h}\right)$ :

$$
\frac{\mathrm{a}_{\mathrm{th}}}{\cos \frac{\pi a_{t h}}{2 W}}=\frac{\mathrm{K}_{\mathrm{th}}^{2}}{\sigma_{\max }^{2} \pi}
$$

For a given $a_{0}$ the corresponding load where $\Delta K<\Delta K_{\text {th }}$ can also be determined:

$$
\Delta \sigma_{t h}=\frac{\Delta K_{t h}}{\sqrt{\pi a_{0}}} \sqrt{\cos \frac{\pi a_{0}}{2 W}}
$$

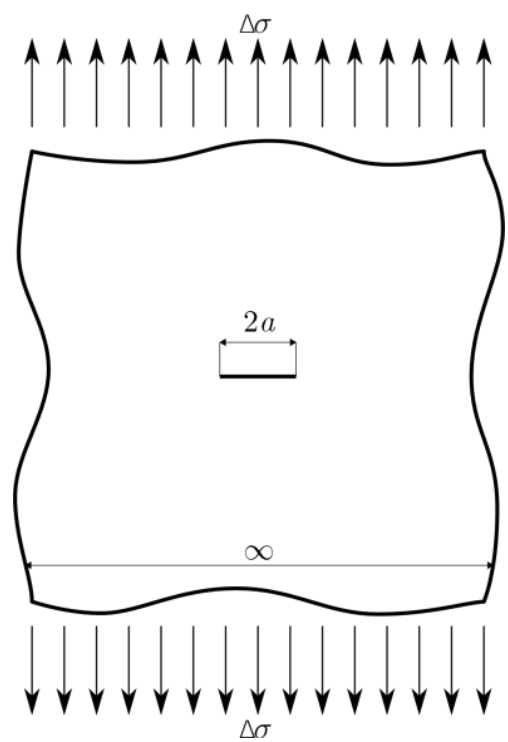

Figure 1: Finite plate under stress loading

\section{Results}

\subsection{Finite plate}

Initially, the influence of $W$ on $a_{c}$ was checked and is displayed in Figure 2, showing that for low $K_{c}$, relative to $\Delta \sigma, W$ has a negligible influence on $a_{c}$, but as $K_{c}$ increases the difference on $a_{c}$ caused by $W$ starts being noticeable. It is also noted that as $W$ is increased the increases on $a_{c}$ resulting from it start being smaller and smaller, for example the difference between $W=$ $1000 \mathrm{~mm}$ and $W=10000 \mathrm{~mm}$ is much smaller than the difference between $W=100 \mathrm{~mm}$ and $W=200 \mathrm{~mm}$. Another conclusion that can be drawn from this figure is that $a_{c}$ tends to reach a plateau corresponding to $W$ as $K_{c} / \Delta \sigma$ increases. 


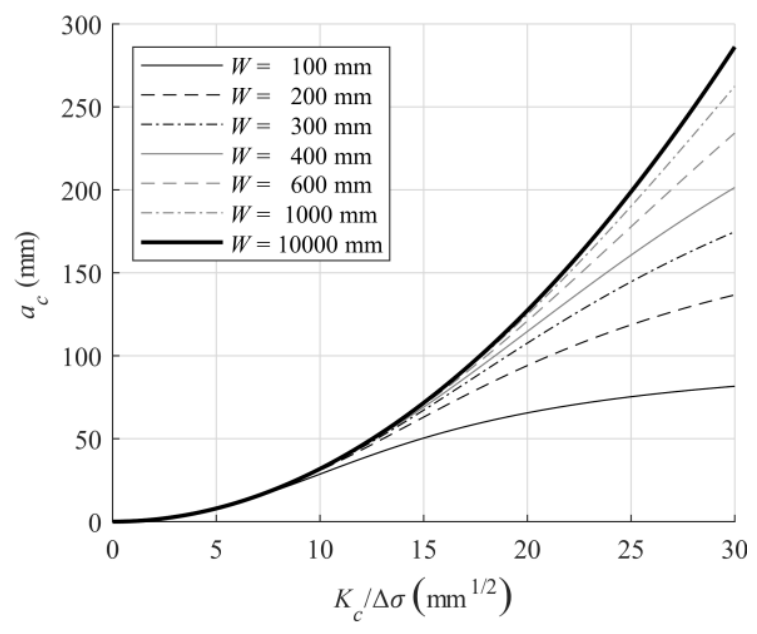

Figure 2: Critical crack length as a function of Kc

In the parametric study conducted for the finite plate case the default values are: $W=100$ $\mathrm{mm} ; \Delta \sigma=100 \mathrm{MPa} ; m=2.5 ; C=1 \times 10^{-11} ; K_{c}=3000 \mathrm{~N} / \mathrm{mm}^{3 / 2} ; a_{0}=10 \mathrm{~mm}$. One at a time, these values were changed to determine their influence on the number of cycles before failure. Figure 3 shows the crack evolution with number of cycles varying $W$ and $\Delta \sigma$, with " $x$ " marking failure caused by reaching $a_{c}$. The $\Delta \sigma$ has a much larger influence than $W$ on the number of cycles before failure and crack evolution. For lower $\Delta \sigma$ changes to it are more significant both in crack evolution and cycles before failure but change $a_{c}$ slightly, when compared to changes in $\Delta \sigma$ for high $\Delta \sigma$. Crack growth tends to start small and then it increases exponentially, which is expected since Paris' law predicts a crack growth rate increase with crack size increase. In Figure $3(\mathrm{a})$, the comparison between $W=10000 \mathrm{~mm}$ and $W=\infty$ also shows that the crack increment used to perform the integration by summation is sufficiently small that the error due to this approximation is also small.

The analysis of Figure 4 shows that $m$ has the largest influence in $N_{f}$ when compared to the other parameters for high loads, low cycle life. However, $C$ also greatly influences fatigue life, and for lower loads it is more influential than $m$. For both $m$ and $C$ smaller values result in higher $N_{f}$, and the inverse happens for higher values of $m$ and $C$.

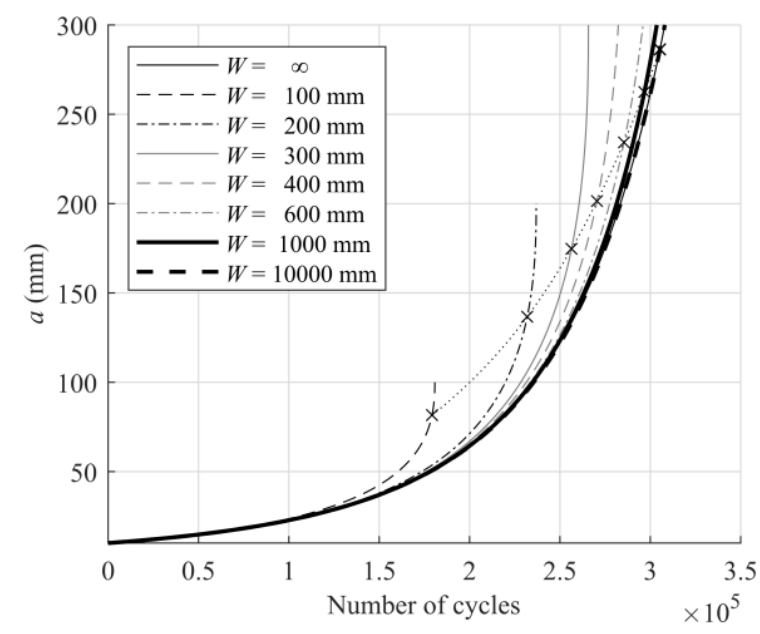

(a)

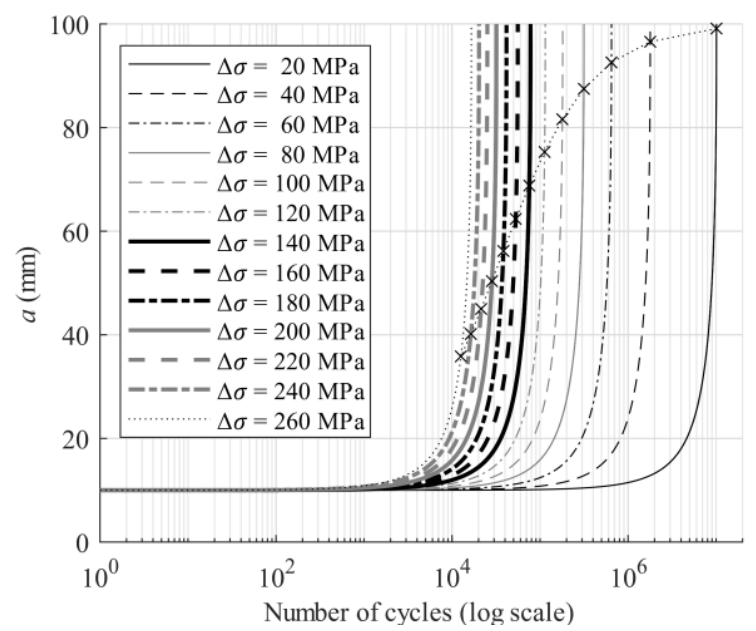

(b)

Figure 3: Crack evolution for different (a) W, (b) $\Delta \sigma$ 


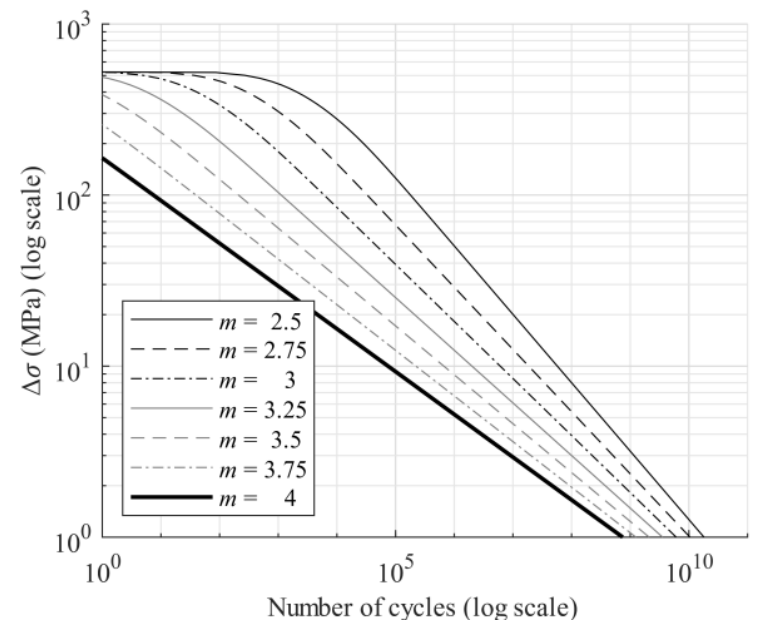

(a)

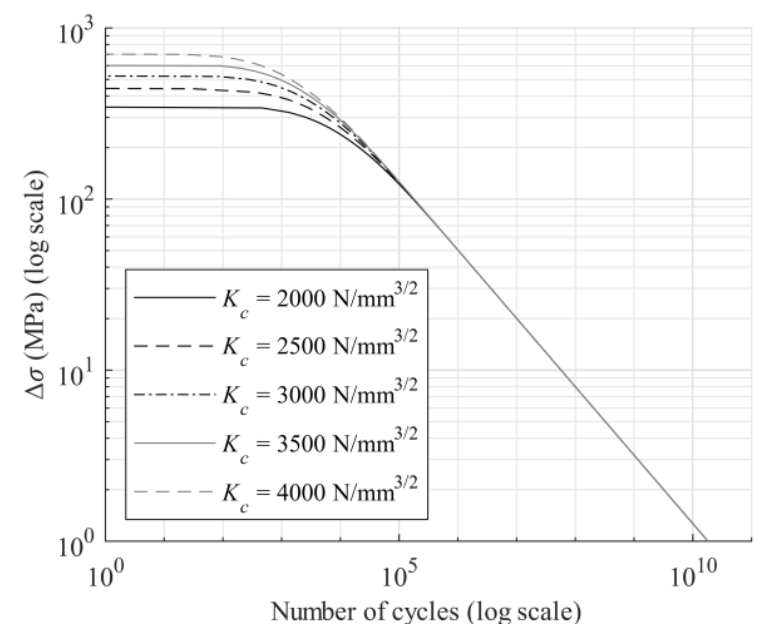

(c)

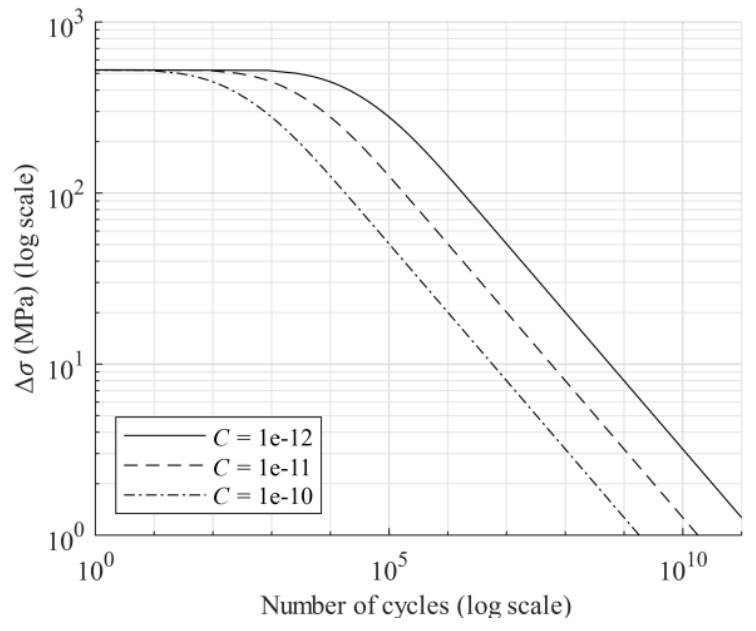

(b)

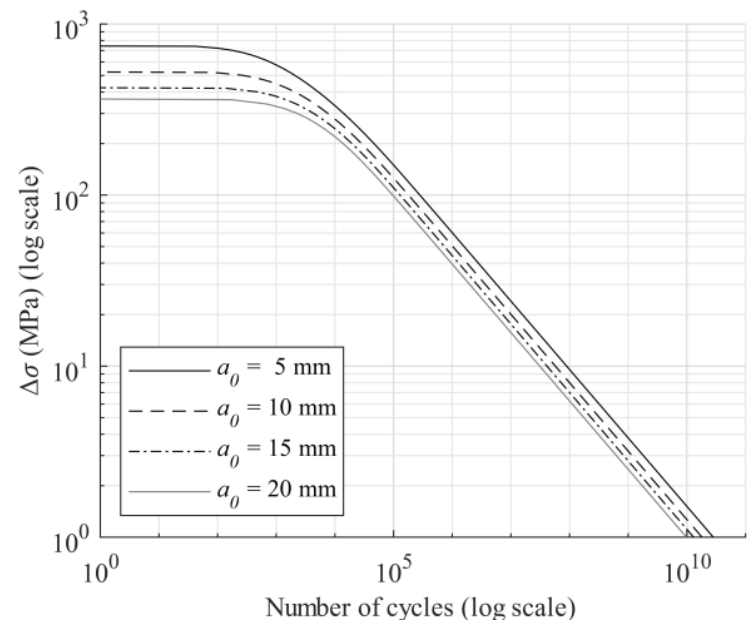

(d)

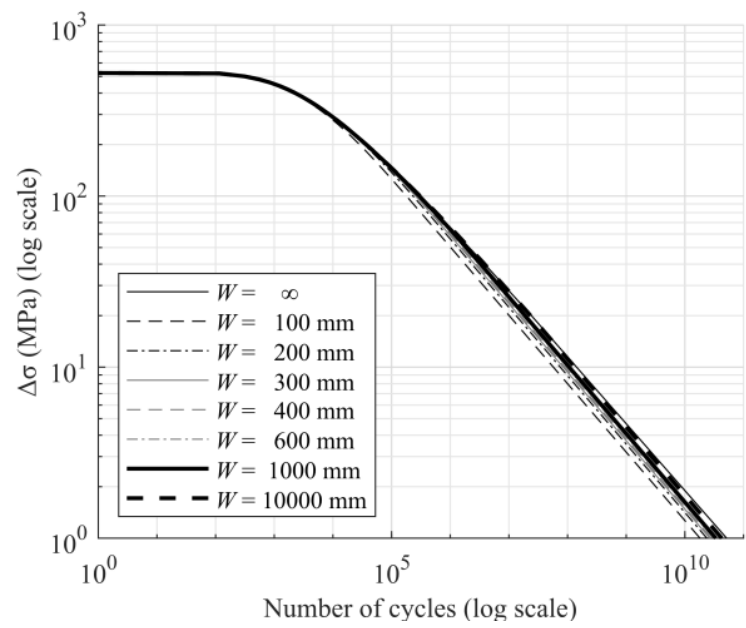

(e)

Figure 4: Number of cycles until failure as a function of the stress range, with varying (a) $\mathrm{m}$, (b) $\mathrm{m},(\mathrm{c}) \mathrm{Kc}$, (d) a0, (e) W

There is a $\Delta \sigma$ plateau, if $\Delta \sigma$ is higher than that the plateau supports no cycles, this plateau is increased by increasing $K_{c}$ or decreasing $a_{0}$, and it is decreased by the opposite changes to $K c$ and $a_{0}$. This is due to Equation (4), by replacing $a_{c}$ with $a_{0}$ and rearranging it is possible to determine the $\Delta \sigma$ at which the plateau occurs: 


$$
\Delta \sigma_{p}=\frac{K_{c} \sqrt{\cos \frac{\pi a_{0}}{2 W}}}{\sqrt{\pi a_{0}}}
$$

which corresponds to a load that makes the crack start propagating unstably, causing failure, after just one load. $K_{c}$ has an influence in the number of cycles before failing only for $\Delta \sigma>100$ $\mathrm{MPa}$, having minimal effect for loads lower than that. Figure 4 shows the effect of plate width, which is much smaller than the effect of the other parameters, and for high loads it is approximately non-existent.

\subsection{Finite plate considering $K_{\text {th }}$ and general yielding}

In the parametric study conducted for the current case the default values are: $W=100 \mathrm{~mm}$; $\Delta \sigma=100 \mathrm{MPa} ; m=2.5 ; C=1 \times 10^{-11} ; K_{c}=3000 \mathrm{~N} / \mathrm{mm}^{3 / 2} ; a_{0}=10 \mathrm{~mm} ; K_{t h}=400 \mathrm{~N} / \mathrm{mm}^{3 / 2} ; \sigma_{Y}=$ $300 \mathrm{MPa}$. One at a time, these values were changed to determine their influence on the number of cycles before failure.

Figure 5 shows how $a_{c}, a_{Y}$ and $a_{t h}$, evolve depending on the load applied, for the default values. For a given $\Delta \sigma$ failure will occur due to $a_{c}$ or $a_{Y}$ being reached, whichever is lower. For $W=100$ $\mathrm{mm}$ failure will always occur due to $a_{r}$ being reached, however, for larger $W$ failure occurs due to $a_{Y}$ being reached for low loads, then it occurs due to $a_{c}$ being reached, and finally it occurs again due to $a_{Y}$ being reached. As $W$ increases the region where it occurs due to $a_{c}$ being reached also increases. Regarding $a_{t h}$, it is approximately the same for all $W$ tested when the $\Delta \sigma>50 \mathrm{MPa}$ if $a_{0}<10 \mathrm{~mm}$ the crack would not grow for $\Delta \sigma<70 \mathrm{MPa}$, regardless of $W$.

Figure 6 shows the crack evolution with number of cycles varying $W$ and $\Delta \sigma$, with " $x$ " marking failure caused by reaching $a_{c}$, "o" marks failure caused by general yielding and diamond marks the threshold crack length. By testing different $W$ it is visible that for small $W$ failure is generally due to general yielding, but as $W$ increases the cause of failure starts being $K_{c}$ instead. For the default $W=100 \mathrm{~mm}$ failure is always due to general yielding regardless of $\Delta \sigma$ applied to the plate, it is also interesting to note that for low $\Delta \sigma$ the crack would not propagate according to Paris' law using $a_{0}=10 \mathrm{~mm}$, but if the initial crack was higher it would obey Paris' law, as was expected from Figure 6 . For $W=300 \mathrm{~mm}$ failure starts out by being due to yield for low loads, but as they increase failure is due to $K_{c}$ being exceeded, this change from one criterion to the other happens at $\Delta \sigma \approx 60 \mathrm{MPa}$, however, failure is again dictated by general yielding when the loads start approaching $\sigma_{Y}$, as was expected from Figure 6.

The parametric tests presented in Figure 7 are similar to Figure 4 of the previous section, however the $\Delta \sigma$ plateau occurs at a lower level because for the default $\sigma_{Y}=300 \mathrm{MPa}$ the $\Delta \sigma_{p}$ that can be determined using yield is lower than the plateau that can be determined using $K_{c}$. It is also important to note that in the places where the curves bellow the dashed line $\Delta K<K_{\text {th }}$ meaning that crack growth does not obey Paris' law, generally growing more slowly than it.

Figure 7 (c) shows clearly that for the default parameters chosen failure is determined by general yielding and changing $K_{c}$ does not affect the number of cycles before failure. Given that, different $\sigma_{Y}$ were tested to determine at which point failure would be determined by $K_{c}$ in Figure $7(\mathrm{f})$, from that it was concluded that failure starts being solely determined by $K_{c}$ regardless of $\Delta \sigma$ when $\sigma_{Y}>600 \mathrm{MPa}$ in this case, however if $K_{c}$ was lower this would happen sooner and the inverse would be verified if $K_{c}$ was higher. 


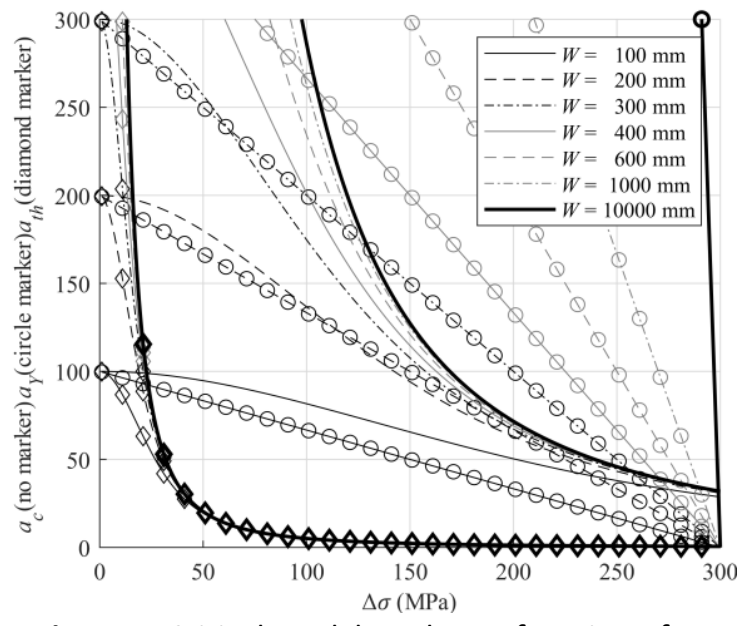

Figure 5: Critical crack length as a function of Kc

In this case there is also a $\Delta \sigma$ plateau, which can either be caused by $K_{c}$ being reached at $a_{0}$, or be caused by general yielding at $a_{0}$. In the first case Equation (12) can also be used, in the second case the following equation is used:

$$
\Delta \sigma_{p}=\frac{W \sigma_{Y}-a_{0} \sigma_{Y}}{W}
$$

whichever of those two values is lower will be the $\Delta \sigma_{p}$.

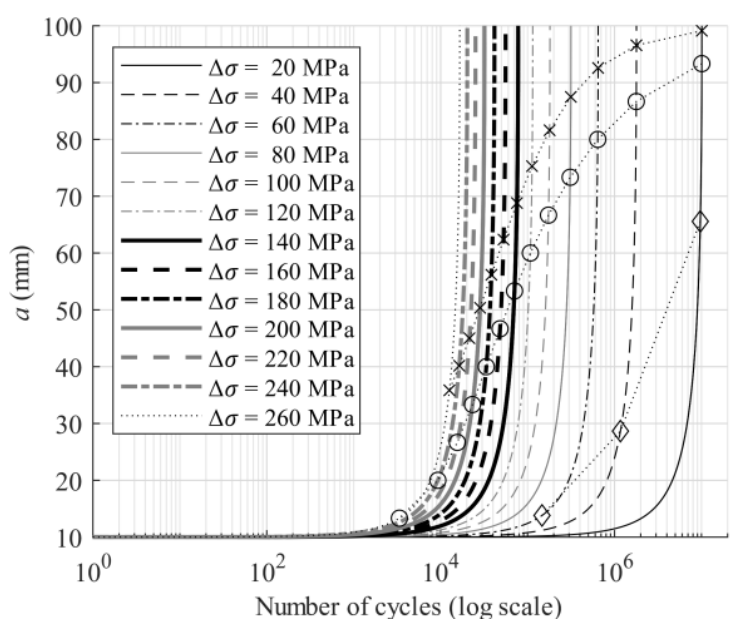

(a)

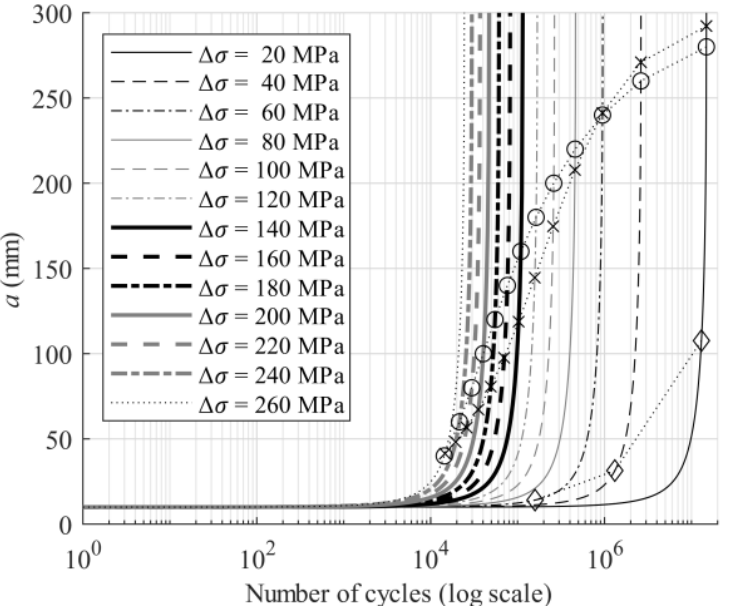

(b)

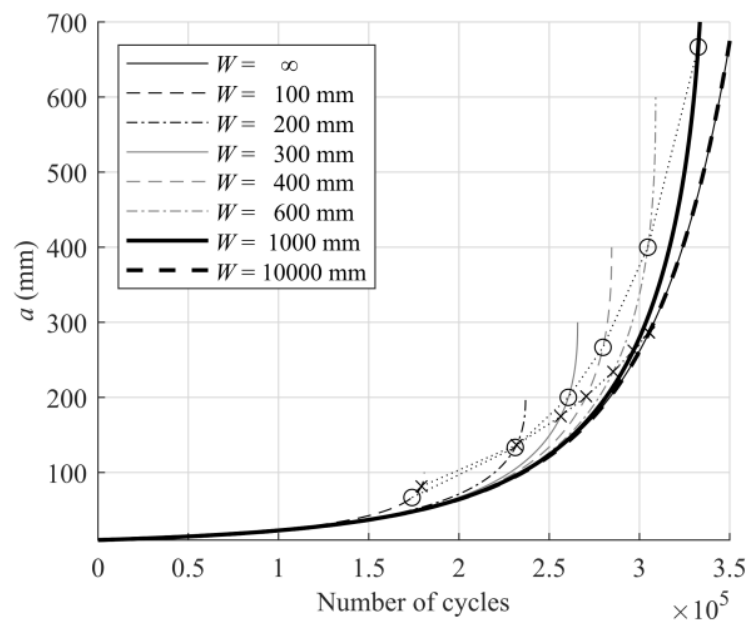

(c)

Figure 6: Crack evolution for different (a) $W$, (b) $\Delta \sigma$ with $W=100$, (c) $\Delta \sigma$ with $W=$ 300 


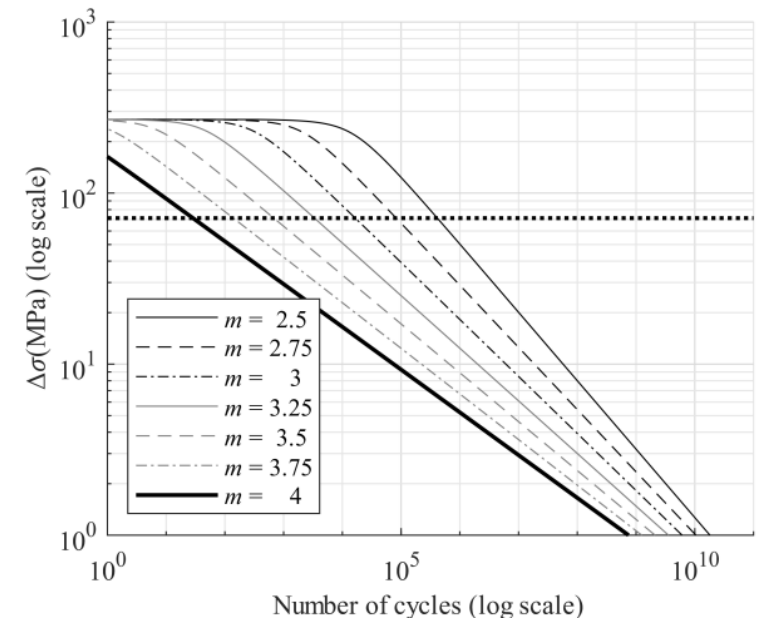

(a)

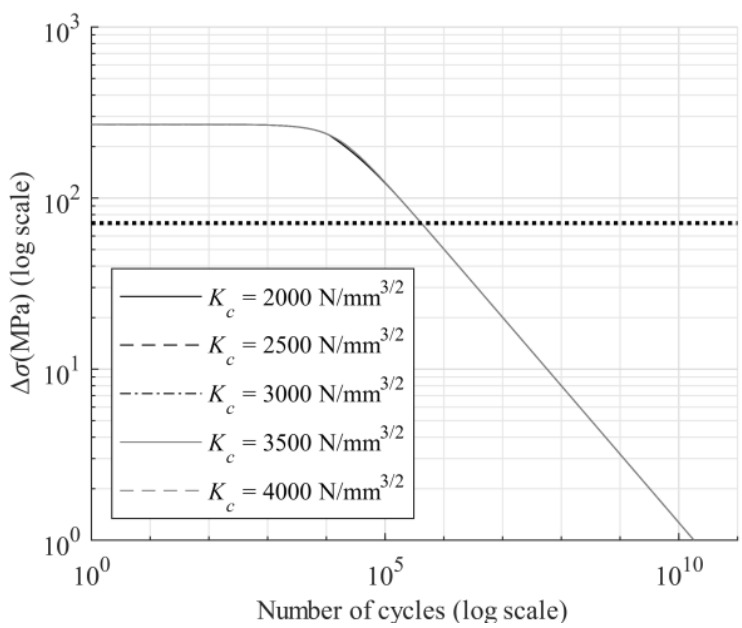

(c)

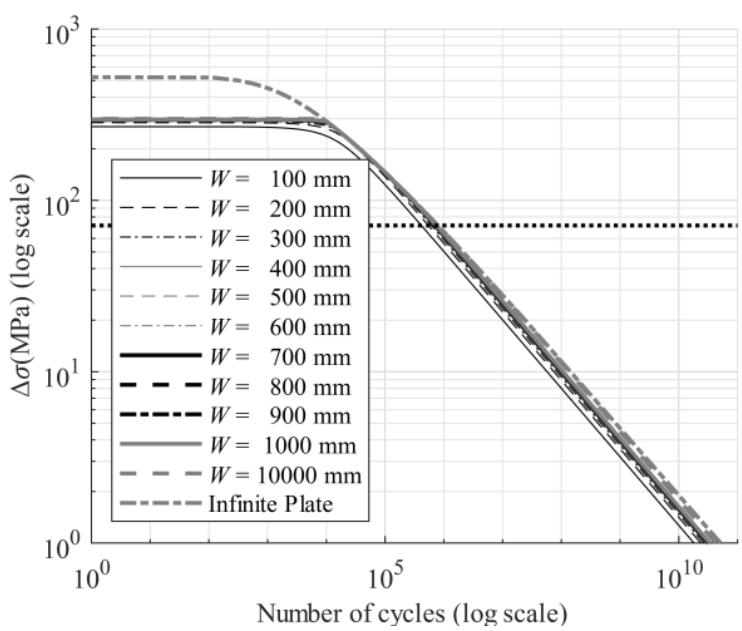

(e)

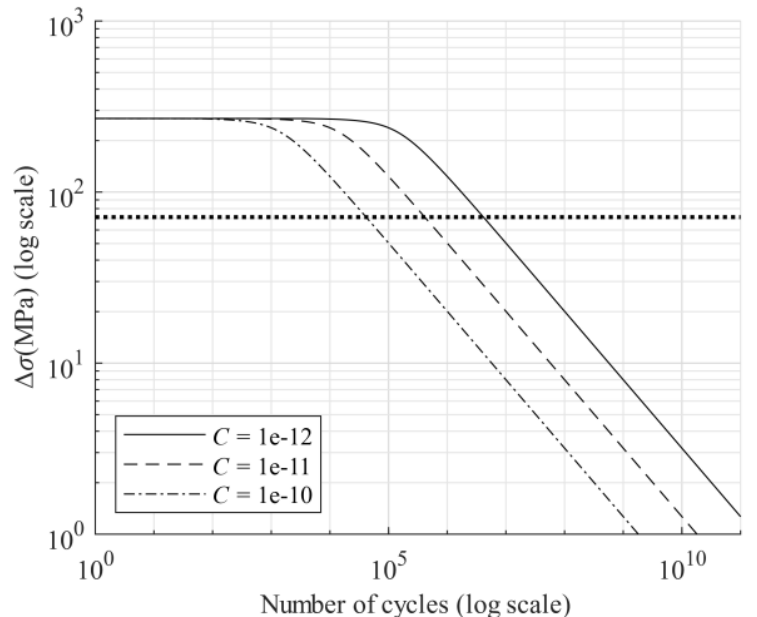

(b)

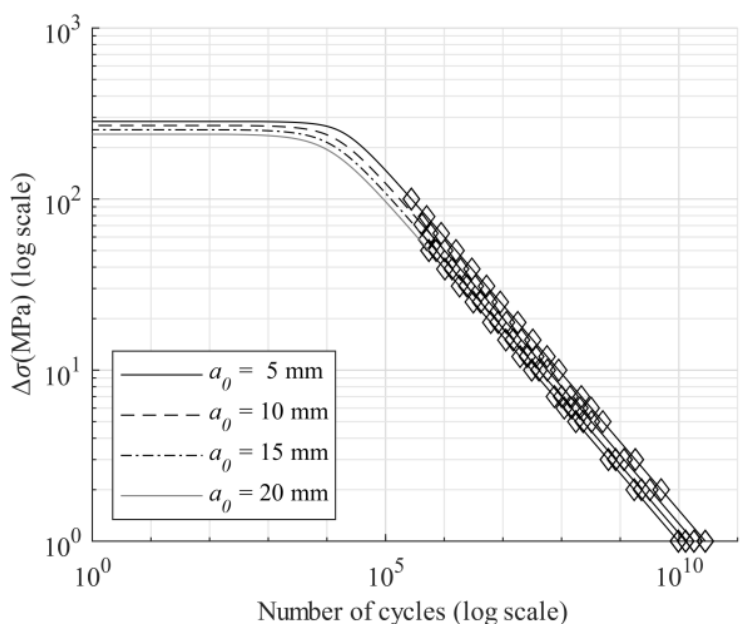

(d)

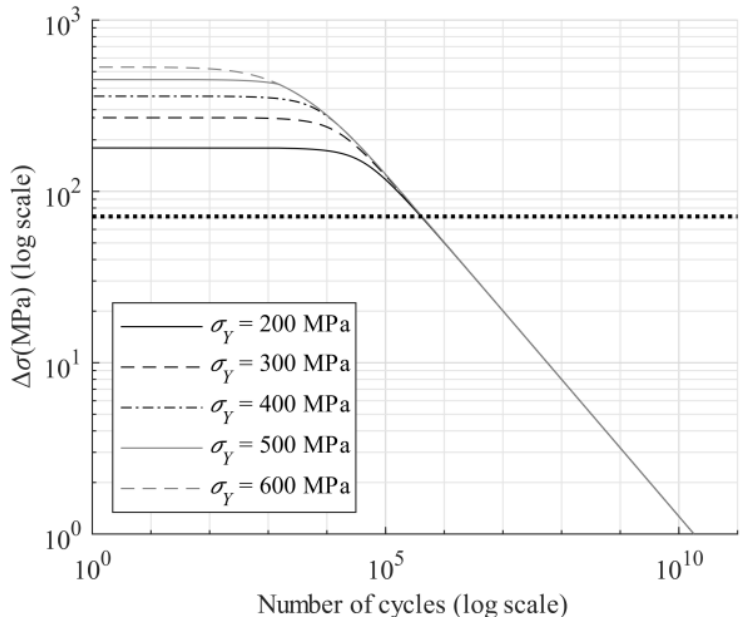

(f)

Figure 7: Number of cycles until failure as a function of the stress range, with varying (a) $m,(b) m,(c) K c,(d) a 0,(e) W,(f) \sigma Y$

\section{Conclusions}

This work presents a parametric study of fatigue crack propagation and resulting life for a plate with finite width and a central crack. The study shows that the Paris' law parameters $m$ and $C$ have a significant effect when compared to $K_{c}$ and $a_{0}$, which have a significantly smaller 
effect. Interestingly $W$ does not have a significant effect on life, at least when $W / a_{0} \geq 10$ and only failure due to crack growth is considered, so the infinite plate, which is much simpler mathematically, could be used to approximate the finite plate without affecting life predictions much. By considering general yielding as a failure criterion, it was verified that with high stresses failure could either be limited by $\sigma_{Y}$ or $K_{c}$ depending on their values. However for low loads, $\Delta \sigma<100 \mathrm{MPa}$, there is not a big difference regardless on $K_{c}$ or $\sigma_{\gamma}$.

The parametric study performed shows that to extend the fatigue life of a given component it is important that the material chosen has a low $m$ and $C$, the Paris' law parameters, and for components subjected to relatively high stresses it is also important to ensure that $\sigma_{Y}$ is sufficiently high to prevent rupture due to general yielding.

\section{References}

Albert, W. A. J. 1838. "Über Treibseile Am Harz". Archiv Für Mineralogie, Georgnosie Bergbau Und Huttenkunde 10: 215-34.

Bulloch, J. H. 1991. "The influence of mean stress or R-ratio on the fatigue crack threshold characteristics of steels - A review". International Journal of Pressure Vessels and Piping 47, no. 3: 263-92. https://doi.org/10.1016/0308-0161(91)90032-W.

Butler, S., and J. M. M. Rodriguez. 1992. "Implications of fracture-mechanics in engineering design". Engineering Fracture Mechanics 42, no. 2: 339-46. https://doi.org/10.1016/00137944(92)90224-3.

Irwin, G. R. 1957. "Analysis of stresses and strains near the end of a crack traversing a plate". Journal of Applied Mechanics 24: 351-69.

Lawson, L., E. Y. Chen, and M. Meshii. 1999. "Near-threshold fatigue: A review". International Journal of Fatigue 21: S15-S34. https://doi.org/10.1016/S0142-1123(99)00053-5.

Mínguez, J. M., and J. Vogwell. 1993. "Safe life of cracked structural elements". Engineering Fracture Mechanics 46, no. 1: 143-50. https://doi.org/10.1016/0013-7944(93)90311-F.

Paris, P. C., M. P. Gomez, and W. E. Anderson. 1961. "A rational analytic theory of fatigue". The Trend in Engineering 13: 9-14.

Paris, P., and F. Erdogan. 1963. "A critical analysis of crack propagation laws". Journal of Fluids Engineering 85, no. 4: 528-33. https://doi.org/10.1115/1.3656900.

Ritchie, R. O. 1979. "Near-threshold fatigue-crack propagation in steels". International Metals Reviews 24, no. 1: 205-30. https://doi.org/10.1179/imtr.1979.24.1.205.

Schütz, W. 1996. "A history of fatigue". Engineering Fracture Mechanics 54, no. 2: 263-300. https://doi.org/10.1016/0013-7944(95)00178-6.

Stephens, R., A. Fatemi, R. R. Stephens, and H. Fuchs. 2001. Metal fatigue in engineering. $2^{\text {nd }}$ ed. John Wiley \& Sons.

Wöhler, A. 1860. "Versuche zur ermittlung der auf die eisenbahnwagenachsen einwirkenden kräfte und die widerstandsfähigkeit der wagen-achsen". Zeitschrift Für Bauwesen 10 no. 1860: 583-614.

\section{Acknowledgments}

The authors acknowledge the funding provided by Ministério da Ciência, Tecnologia e Ensino Superior - Fundação para a Ciência e a Tecnologia (Portugal), under project funding POCI-010145-FEDER-028351 and SFRH/BD/147628/2019. Additionally, the authors acknowledge the funding provided by LAETA, under project UIDB/50022/2020. 